\title{
Conhecimento tecnológico dos professores de Matemática sobre quadros interativos segundo as políticas públicas de formação contínua ${ }^{1}$
}

Patricia Alexandra da Silva Ribeiro Sampaio ${ }^{a}$

\section{Resumo}

O Plano Tecnológico da Educação foi o maior programa de modernização tecnológica das escolas portuguesas e era composto por três eixos: Tecnologia, Conteúdos e Formação. Apresenta-se um estudo sobre o impacto destas políticas públicas nas práticas dos docentes sobre o uso de quadros interativos (QI). Aplicou-se um questionário a 453 docentes de Matemática de Portugal e concluiu-se que estes consideram que possuem um nível bom em TIC, usam-nas com frequência nas aulas, consideram ser detentores de conhecimento tecnológico sobre QI, mas ainda é visível uma dispersão de uso. Os docentes sentem maior necessidade de aperfeiçoamento da capacidade de usar os QI para proporcionar aos alunos diferentes oportunidades de aprendizagem e praticar Matemática. A frequência em formação contínua no âmbito destas políticas trouxe melhorias significativas no conhecimento tecnológico dos docentes sobre esta ferramenta, mas ainda há um longo caminho a percorrer para o desenvolvimento do TPACK Matemático.

Palavras-chave: Quadro interativo. TPACK matemático. Formação contínua. Políticas públicas.

\section{Introdução}

O Plano Tecnológico da Educação (PTE), programa de modernização tecnológica das escolas portuguesas, visava constituir uma viragem decisiva na utilização generalizada das tecnologias de informação e comunicação (TIC) no processo de

\footnotetext{
1 Artigo redigido no âmbito de uma bolsa de doutoramento SFRH/BD/71323/2010 financiada pela Fundação para a Ciência e Tecnologia (Portugal).

a Universidade do Minho. Braga, Portugal.
} 
ensino/aprendizagem. Pretendia que a Escola se tornasse num espaço interativo e de partilha de conhecimento, certificando as competências TIC de docentes, estudantes e funcionários e preparando os alunos para a sociedade do conhecimento. O PTE era composto por três eixos de atuação: Tecnologia, Conteúdos e Formação. Aprovado em setembro de 2007 pelo Governo Português, em julho de 2009, foi criado o sistema de formação e de certificação em competências TIC para docentes, que foi aplicado ao longo de quatro anos, até 2013.

Neste artigo, faz-se uma breve referência ao propósito da política pública do PTE, expondo que o impacto das TIC na Escola está diretamente relacionado com o modo como é utilizada pelos docentes com os alunos e não com a tecnologia em si (KOEHLER; MISHRA, 2005; SAMPAIO; COUTINHO, 2012) e refere-se que o TPACK (conhecimento tecnológico e pedagógico do conteúdo) é um referencial teórico relevante para o ensino da Matemática que deve ser desenvolvido no processo de ensino/aprendizagem e que a formação contínua de docentes deve ajudá-los a tornarem-se bem informados sobre a tecnologia, de forma a os desafiar a integrá-la no ensino (NIESS, 2006).

Através da política pública do PTE estava previsto assegurar um quadro interativo (QI) em cada três salas de aula e a formação da maioria dos docentes das escolas públicas portuguesas. Apresenta-se um estudo sobre o impacto do PTE nas práticas letivas dos docentes de Matemática sobre o uso de QI em contexto de sala de aula.

\section{Integração das TIC no Ensino de Matemática}

O governo português criou o PTE que visava a integração da tecnologia nas escolas públicas e estruturava-se segundo três eixos temáticos de intervenção: tecnologia, conteúdos e formação. Em 2009, foram produzidas as condições normativas para a concretização do programa de formação e de certificação de competências TIC e pretendia envolver $90,0 \%$ dos docentes das escolas públicas, ao longo de quatro anos (2010-2013). Através do PTE as escolas foram modernizadas tecnologicamente e foi proporcionada formação contínua aos docentes. Deste modo, nas escolas de hoje encontra-se disponível um conjunto variado de recursos para facilitar a implementação de metodologias de ensino apoiadas nas TIC.

Os QI são um dos recursos alvo do PTE, tendo o governo pretendido instalar 5.613 QI com videoprojetor nas escolas e fornecer formação à maioria dos docentes sobre o seu uso tecnológico e pedagógico. Estas ferramentas tecnológicas permitem a utilização simultânea de imagens, vídeos, tabelas, gráficos, folhas de cálculo, diferentes softwares, criar anotações em direto sobre documentos, gravar todos os registos da aula... "A rentabilização desse recurso permite ao professor 
realizar uma gestão mais eficiente do tempo de aula com propostas desafiadoras e enriquecedoras para os alunos" (SAMPAIO; COUTINHO, 2013a, p. 744). Não obstante, vários estudos salientam que o impacto das TIC no processo de ensino/aprendizagem está diretamente relacionado com a forma como são utilizadas pelos docentes nas suas práticas letivas e não com a tecnologia em si (KOEHLER; MISHRA, 2005; SAMPAIO; COUTINHO, 2012).

O TPACK é um referencial teórico que explica as complexas interligações entre conteúdo, pedagogia e tecnologia (KOEHLER; MISHRA, 2005). E, em particular, no caso da Matemática "o modelo de desenvolvimento do Mathematics Tpack fornece um referencial dinâmico para examinar o conhecimento que o professor [de Matemática] precisa ter para lidar com a complexidade do ato de ensinar [Matemática] com tecnologia" (PALIS, 2010, p. 449).

Segundo Mishra e Koehler (2006, p. 1020), "a base deste quadro teórico é o entendimento que o ensino é uma atividade extremamente complexa que recorre a diversos tipos de conhecimentos". Ensinar eficazmente com tecnologia requer uma compreensão de cada um destes conhecimentos, mas também e, essencialmente, de todas as relações de reforço mútuo entre tecnologia, conteúdo e pedagogia (Figura).

Um professor só conseguirá tomar decisões fundamentadas de integração das tecnologias no processo de ensino/aprendizagem se souber manusear as tecnologias, numa área curricular, através de uma estratégia pedagógica, num certo contexto educativo, como promoção da construção do conhecimento do aluno, para contribuir para o alcance de um determinado objetivo educacional (SAMPAIO; COUTINHO, 2013b).

\section{Quadros Interativos}

Os QI começaram a ser produzidos em 1991, mas só a partir do século XXI é que a sua difusão começou a ocorrer no campo da educação. Existem basicamente dois tipos de QI de acordo com o controlo do computador: os que funcionam através do toque das mãos ou de qualquer objeto e os que funcionam com o uso de canetas interativas. Não obstante, todos têm em comum o cruzamento de várias funcionalidades numa só ferramenta: inserção/edição/visualização/captura de texto, imagens, som e vídeos; construção/manipulação/modificação de documentos; partilha em tempo real; acesso à Internet; manipulação de diferentes softwares; salvar em diferentes formatos... 


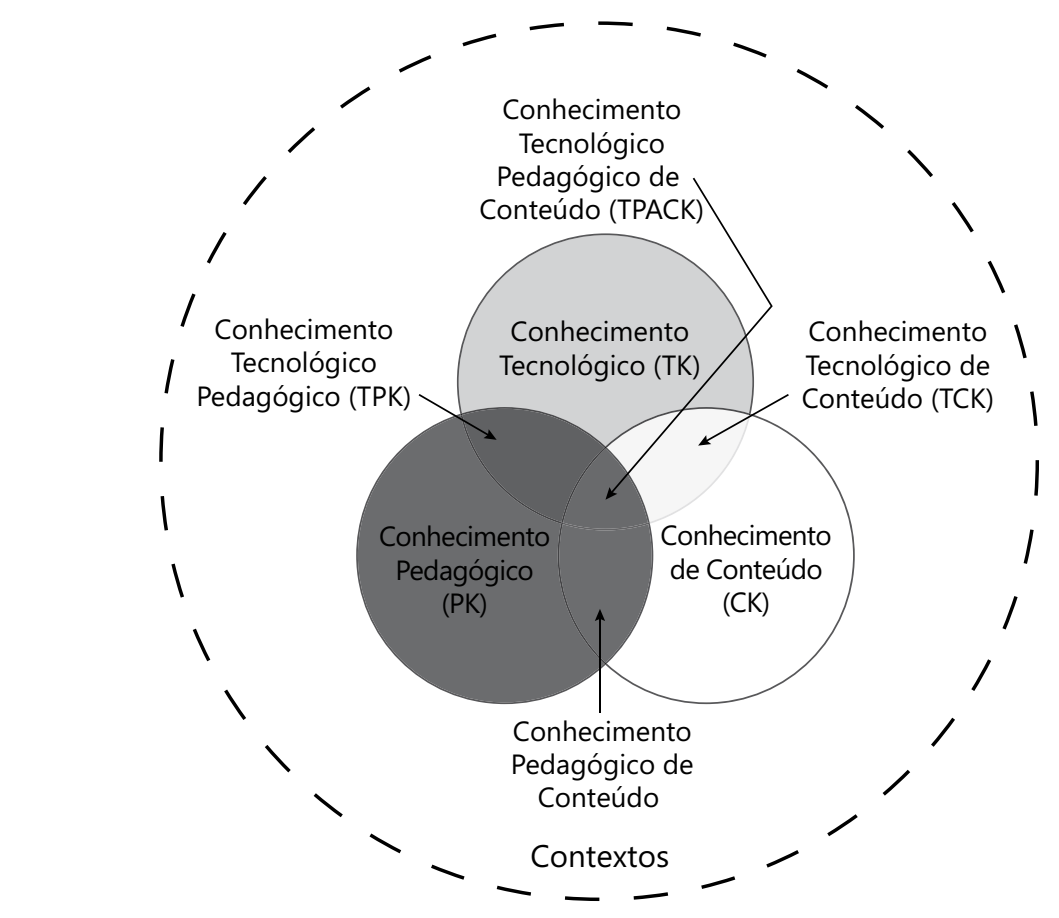

Fonte: http://tpack.org (2012).

Figura. O referencial TPACK e os seus componentes do conhecimento.

Um QI é um dispositivo que é ligado a um computador e o ecrã do computador é projetado para o quadro através de um videoprojector, mas para além da sua visualização também existe interação, através do dedo ou de uma caneta, diretamente no quadro. Cada QI apresenta um software específico, embora a sua utilização se assemelhe em termos de intuição. O professor tem acesso a uma galeria de recursos bastante vasta e, assim, pode preparar, previamente, as suas aulas com o software específico do QI. Permite as utilizações mais variadas, como construir e arrastar objetos, escrever e reconhecer letra manuscrita, ativar hiperligações, utilizar simultaneamente texto, imagem e vídeo... A sua conexão ao computador permite que o professor tenha acesso direto a um conjunto de recursos disponíveis na Internet ou nas suas pastas pessoais, possibilitando um leque muito variado de propostas de atividades em sala de aula. Salienta-se ainda que a utilização do QI não se restringe ao uso do software específico do próprio quadro, pois podem ser usados todos os outros recursos disponíveis no computador como o processador de texto, a folha de cálculo, a plataforma da escola, o blogue do professor, software de geometria dinâmica... 
As potencialidades educativas desta ferramenta foram compiladas por Sampaio e Coutinho (2013a) numa avaliação que realizaram através de diversas pesquisas efetuadas em diferentes países, tendo concluído que os QI são atraentes, adequam-se ao ensino da turma como um grupo, permitem a integração de diferentes recursos, estimulam a interatividades, aceleram o ritmo das aulas, permitem a ligação à Internet, motivam os alunos, permitem salvar e reutilizar materiais que foram criados/anotados na aula. Não obstante, referiram alguns inconvenientes de natureza prática ou logística.

Em países como os Estados Unidos da América, o Reino Unido, a Austrália e a França os QI já são usados no ensino há alguns anos, no entanto, em Portugal, a sua utilização surgiu praticamente com o PTE.

\section{Método}

Através deste estudo pretendeu-se responder às seguintes questões de investigação:

1. Qual o grau de competência que os docentes de Matemática consideram ter na utilização das TIC na prática letiva?

2. Com que frequência utilizam os QI nas suas aulas?

3. Qual a sua autoperceção sobre o conhecimento tecnológico na utilização de QI?

4. A frequência de formação, no âmbito das políticas públicas do PTE, provocou diferenças no conhecimento tecnológico em QI dos professores de Matemática?

\subsection{Amostra}

Os docentes de Matemática em Portugal dividem-se em dois grupos de recrutamento de acordo com o nível de ensino: os do grupo 230 que lecionam ao $2^{\circ}$ ciclo do ensino básico (CEB) e os do grupo 500 que lecionam ao $3^{\circ} \mathrm{CEB}$ e ao secundário. Participaram $62,3 \%$ do grupo 500 e $37,7 \%$ do grupo 230 , tendo $7,8 \%$ menos de 10 anos de serviço, $43,7 \%$ entre 10 e $20,46,4 \%$ entre 20 e 30 e $12,1 \%$ entre 30 e 40 anos de serviço. A sua distribuição pelas escolas de Portugal continental foi a seguinte: 37,1\% trabalha na Direção de Serviços da Região (DSR) Norte, 20,1\% na do Centro, 24,9\% na de Lisboa e Vale do Tejo, 8,4\% na do Alentejo e 9,5\% na do Algarve. Participaram no total 453 docentes de Matemática do ensino público, em Portugal continental, $75,9 \%$ do sexo feminino e $24,1 \%$ do sexo masculino. 
Estes dados podem ser considerados semelhantes ao último estudo publicado pela Direção-Geral de Estatísticas da Educação e Ciência (DGEEC, 2014), relativo ao ano letivo 2012/2013, sobre as estatísticas da educação, que contabilizou 76,4\% docentes de Matemática do sexo feminino e 23,6\% do sexo masculino em Portugal continental, sendo $41,8 \%$ do grupo 230 e $58,2 \%$ do grupo 500 , distribuídos pelas DSR da seguinte forma: $37,6 \%$ na do Norte, $22,3 \%$ na do Centro, $26,9 \%$ na de Lisboa e Vale do Tejo, 8,1\% na do Alentejo e 5,1\% na do Algarve.

\subsection{Instrumento}

Aplicou-se um questionário destinado aos docentes de Matemática sobre o uso dos QI na sala de aula composto por quatro questões relativas à amostra: sexo, grupo de recrutamento, anos de serviço e DSR da escola onde trabalha. O questionário era composto por três questões em escala relacionadas com o uso das TIC: uma relacionada com a frequência de uso das TIC na sala de aula (as respostas variavam entre 1 - nunca e 5 - sempre); outra relacionada com o grau de competência na utilização das TIC na prática docente (as respostas variavam entre 1 - muito mau e 5 - muito bom) e a terceira estava relacionada com a frequência de uso do QI nas aulas (as respostas variavam entre 1 - nunca e 5 - sempre).

O questionário pretendia avaliar os diferentes tipos de conhecimento tecnológico associados ao TPACK Matemático no caso particular do QI. Era composto por sete itens em escala: um sobre TK, dois sobre TPK, um sobre TCK e três sobre TPACK, em que o conteúdo estava diretamente relacionado com a Matemática (Quadro 1). Os sete itens de opinião respeitaram uma escala de Likert de cinco pontos: 1 - discordo totalmente, 2 - discordo, 3 - não concordo nem discordo, 4 - concordo e 5 - concordo totalmente.

Pretendeu-se ainda recolher informação relativa à formação que os docentes tinham recebido sobre QI no âmbito das políticas públicas do PTE. Deste modo, realizou-se uma questão em que se ficava a conhecer se os docentes tinham frequentado formação e, no caso afirmativo, se tinha sido apenas uma ou mais que uma. No sentido de tornar o questionário mais completo, este era composto por uma questão aberta em que era solicitado aos inquiridos que comentassem a afirmação "Consigo adaptar o uso das tecnologias que aprendi na formação [realizada no âmbito do PTE] a diferentes atividades de ensino de Matemática".

\subsection{Análise Estatística}

A recolha de dados realizou-se durante os meses de maio, junho e julho de 2014 através do Google Drive, já que o questionário foi disponibilizado on-line através 
Quadro 1. Itens do questionário relacionados com o conhecimento tecnológico do TPACK Matemático pelo uso de QI.

\begin{tabular}{|c|c|}
\hline $\begin{array}{l}\text { Conhecimento } \\
\text { tecnológico }\end{array}$ & Item \\
\hline TK & $\begin{array}{l}\text { Possuo as habilidades técnicas que necessito para usar } \\
\text { ferramentas de QI. }\end{array}$ \\
\hline \multirow{2}{*}{ TPK } & $\begin{array}{l}1 \text { Sou capaz de ensinar os meus alunos a usarem as ferramentas } \\
\text { de QI. }\end{array}$ \\
\hline & $\begin{array}{l}2 \text { Sou capaz de integrar o uso de QI na aprendizagem } \\
\text { dos alunos. }\end{array}$ \\
\hline TCK & $\begin{array}{c}\text { Consigo usar QI para criar representações eficazes de conteúdo } \\
\text { matemático. }\end{array}$ \\
\hline \multirow{3}{*}{ TPACK } & $\begin{array}{c}1 \text { Entendo como as potencialidades e as limitações dos } \\
\text { QI podem influenciar a aprendizagem de Matemática dos } \\
\text { meus alunos. }\end{array}$ \\
\hline & $\begin{array}{l}2 \text { Sei como usar as ferramentas de QI para proporcionar } \\
\text { aos alunos diferentes oportunidades de aprendizagem e } \\
\text { praticar Matemática. }\end{array}$ \\
\hline & $\begin{array}{l}3 \text { Consigo planear aulas que combinam adequadamente } \\
\text { Matemática com o uso pedagógico do QI. }\end{array}$ \\
\hline
\end{tabular}

QI: quadro interativos; TK: Conhecimento Tecnológico; TPK: Conhecimento Tecnológico Pedagógico; TCK: Conhecimento Tecnológico de Conteúdo; TPACK: Conhecimento Tecnológico Pedagógico de Conteúdo.

Fonte: Dados da pesquisa (2015).

da ajuda dos Centros de Formação de Professores de Portugal continental que o reencaminharam aos docentes de Matemática das escolas inscritas neles. A sua aplicação era individual, anónima e tinha uma duração esperada de 5 minutos. Utilizaram-se estatísticas descritivas como a média e o desvio-padrão (DP) para os subdomínios do TPACK. Para comparar as médias obtidas de acordo com a frequência em formação contínua, foi utilizado o teste não paramétrico Kruskal-Wallis. As análises foram realizadas via SPSS 22.

\section{Resultados}

Aplicou-se um questionário sobre a utilização de QI em contexto de sala de aula a 453 docentes de Matemática de Portugal continental dividido em quatro partes: uma sobre a caraterização do indivíduo, outra sobre o uso das TIC, uma relacionada com o conhecimento tecnológico do TPACK Matemático pelo uso de QI e, finalmente, uma sobre a formação que tinham recebido no âmbito do PTE. 
Relativamente à frequência de uso das TIC por parte dos docentes em contexto de sala de aula obteve-se uma média de 3,48 , com um DP de 0,930 , numa escala de 1 (nunca) a 5 (muito bom) sendo a moda 4 (frequentemente). No caso da perceção de autoeficácia do grau de competência dos docentes na utilização das TIC na prática docente a média é ligeiramente superior e o DP é menor, isto é, obteve-se uma média de 3,78, com um desvio-padrão de 0,774 , numa escala de 1 (muito mau) a 5 (muito bom) sendo a moda 4 (bom). A maioria dos docentes de Matemática $(79,7 \%)$ considera que possui um grau de competência médio ou bom na utilização das TIC na sua prática letiva e 73,7\% utiliza-as nas suas aulas às vezes ou mesmo frequentemente.

Quanto ao uso propriamente dito do QI, os resultados são bastante inferiores aos observados relativamente às tecnologias em geral, verificando-se uma média de 2,62, com um DP de 1,238, numa escala de 1 (nunca) a 5 (sempre), sendo a moda 3 (às vezes). Neste caso, há uma grande dispersão dos resultados não se salientando uma resposta mais frequente: $24,1 \%$ nunca usa os QI nas suas aulas, $23,6 \%$ raramente, $25,4 \%$ às vezes, $20,1 \%$ frequentemente e só $6,8 \%$ sempre.

No sentido de ficar a conhecer se os docentes tinham frequentado formação sobre QI, estes responderam a uma questão, tendo-se obtido que apenas $20,1 \%$ não tinham realizado formação no âmbito dos QI, salientando-se mesmo que 30,9\% tinham feito mais que uma formação sobre QI.

Relativamente à autoperceção de conhecimento tecnológico sobre a utilização de QI pelos docentes de Matemática de acordo com o TPACK Matemático, estes responderam a uma questão em escala de Likert com cinco pontos, composta por sete itens (Quadro 2), verificando-se grandes semelhanças entre as respostas dadas a cada item, destacando-se apenas o item TPK2 como o que apresenta maior percentagem de respostas afirmativas $(70,2 \%)$ e a menor taxa de incerteza $(15,0 \%)$. Deste modo, podemos concluir que os docentes de Matemática consideram que são capazes de integrar o uso de QI na aprendizagem dos alunos. No entanto, quase metade dos professores $(47,7 \%)$ praticamente não os utiliza em contexto de sala de aula.

Quanto às médias obtidas para cada item, também se pode verificar uma semelhança das mesmas (Quadro 3), rondando os 3,5 e de DP 1, não se salientando grandes distinções na autoperceção dos docentes face aos vários tipos de conhecimentos tecnológicos relativamente aos QI. 
Quadro 2. Percentagem de respostas aos itens em escala sobre o conhecimento tecnológico em QI.

\begin{tabular}{|l|c|c|c|c|c|}
\hline & 1-DT & 2-D & 3-NCND & 4-C & 5-CT \\
\hline TK1 & 5,3 & 12,8 & 18,3 & 48,8 & 14,8 \\
\hline TPK1 & 6,2 & 13,2 & 17,0 & 49,9 & 13,7 \\
\hline TPK2 & 4,9 & 9,9 & 15,0 & 53,9 & 16,3 \\
\hline TCK1 & 6,2 & 12,1 & 24,3 & 43,3 & 14,1 \\
\hline TPACK1 & 4,4 & 5,8 & 22,5 & 53,2 & 14,1 \\
\hline TPACK2 & 6,0 & 13,0 & 24,9 & 44,4 & 11,7 \\
\hline TPACK3 & 6,0 & 12,3 & 24,3 & 43,5 & 13,9 \\
\hline
\end{tabular}

QI: quadro interativo; TK: Conhecimento Tecnológico; TPK: Conhecimento Tecnológico Pedagógico; TCK: Conhecimento Tecnológico de Conteúdo; TPACK: Conhecimento Tecnológico Pedagógico de Conteúdo.

Fonte: Dados da pesquisa (2015).

Quadro 3. Estatísticas descritivas dos itens sobre o conhecimento tecnológico em QI.

\begin{tabular}{|l|c|c|c|c|}
\hline & Média \pm DP & Min-Max & Moda & N \\
\hline TK1 & $3,55 \pm 1,058$ & $1-5$ & 4 & 453 \\
\hline TPK1 & $3,52 \pm 1,078$ & $1-5$ & 4 & 453 \\
\hline TPK2 & $3,67 \pm 1,020$ & $1-5$ & 4 & 453 \\
\hline TCK1 & $3,47 \pm 1,071$ & $1-5$ & 4 & 453 \\
\hline TPACK1 & $3,67 \pm 0,941$ & $1-5$ & 4 & 453 \\
\hline TPACK2 & $3,43 \pm 1,049$ & $1-5$ & 4 & 453 \\
\hline TPACK3 & $3,47 \pm 1,065$ & $1-5$ & 4 & 453 \\
\hline
\end{tabular}

QI: quadro interativo; TK: Conhecimento Tecnológico; TPK: Conhecimento Tecnológico Pedagógico; TCK: Conhecimento Tecnológico de Conteúdo; TPACK: Conhecimento Tecnológico Pedagógico de Conteúdo.

Fonte: Dados da pesquisa (2015).

No sentido de saber se a formação realizada sobre QI salienta diferenças nas respostas obtidas pelos docentes sobre o seu conhecimento tecnológico em QI determinaram-se as médias para cada um dos itens de acordo com a frequência na formação (Quadro 4). Pode-se constatar que há um aumento relevante das médias e uma diminuição do DP de cada item de acordo com a 
Quadro 4. Estatísticas descritivas dos itens sobre o conhecimento tecnológico em Q। de acordo com a frequência em formação.

\begin{tabular}{|c|c|c|c|c|c|c|}
\hline & \multicolumn{6}{|c|}{ Frequentou formação no âmbito dos QI } \\
\hline & \multicolumn{2}{|c|}{$\begin{array}{c}\text { Não } \\
(\mathbf{N}=91)\end{array}$} & \multicolumn{2}{|c|}{$\begin{array}{l}\text { Apenas uma } \\
(\mathrm{N}=222)\end{array}$} & \multicolumn{2}{|c|}{$\begin{array}{l}\text { Mais que uma } \\
\quad(N=140)\end{array}$} \\
\hline & Média \pm DP & $\begin{array}{c}95 \% \\
\text { Inter. Conf. } \\
\text { média }\end{array}$ & Média \pm DP & $\begin{array}{c}95 \% \\
\text { Inter. Conf. } \\
\text { média }\end{array}$ & Média \pm DP & $\begin{array}{c}\text { 95\% Inter. } \\
\text { Conf. } \\
\text { média }\end{array}$ \\
\hline TK1 & $2,91 \pm 1,253$ & ] 2,$65 ; 3,17[$ & $3,59 \pm 1,021$ & ] 3,$46 ; 3,72[$ & $3,90 \pm 0,780$ & ]3,77; 4,03[ \\
\hline TPK1 & $2,79 \pm 1,243$ & ]2,53; 3,05[ & $3,59 \pm 1,028$ & ]3,46; 3,73[ & $3,86 \pm 0,789$ & ] 3,$73 ; 4,00[$ \\
\hline TPK2 & $3,12 \pm 1,298$ & ]2,85; 3,39[ & $3,71 \pm 0,936$ & ] 3,$59 ; 3,84[$ & $3,96 \pm 0,785$ & ] 3,$83 ; 4,09[$ \\
\hline TCK1 & $2,91 \pm 1,305$ & ] 2,$64 ; 3,18[$ & $3,44 \pm 0,981$ & ] 3,$31 ; 3,57[$ & $3,88 \pm 0,852$ & ] 3,$74 ; 4,02[$ \\
\hline TPACK 1 & $3,47 \pm 1,177$ & ] 3,$23 ; 3,72[$ & $3,68 \pm 0,862$ & ] 3,$57 ; 3,79[$ & $3,78 \pm 0,874$ & ]3,63; 3,92[ \\
\hline TPACK2 & $2,79 \pm 1,225$ & ]2,54; 3,05[ & $3,47 \pm 0,964$ & ] 3,$34 ; 3,60[$ & $3,78 \pm 0,857$ & ]3,64; 3,92[ \\
\hline TPACK3 & $2,96 \pm 1,282$ & ] 2,$69 ; 3,22[$ & $3,50 \pm 0,969$ & ] 3,$37 ; 3,62[$ & $3,76 \pm 0,934$ & ]3,61; 3,92[ \\
\hline
\end{tabular}

Fonte: Dados da pesquisa (2015).

frequência dos professores na formação ter sido nula, uma ou mais que uma. O TPACK1, relacionado com a autoperceção dos docentes entenderem como as potencialidades e as limitações dos QI podem influenciar a aprendizagem de Matemática dos seus alunos, foi o que manifestou menor alteração com a frequência em formação. O TPK1 relacionado com a ideia que os docentes possuem de si próprios como capazes de ensinar os seus alunos a usarem as ferramentas de QI foi o que manifestou maior alteração com a frequência em apenas uma formação e os itens TK1 e TPACK2 relacionados, respetivamente, com a autoperceção dos docentes como detentores das habilidades técnicas que necessitam para usarem ferramentas de QI, e sentirem-se capazes de usarem essas ferramentas para proporcionar aos alunos diferentes oportunidades de aprendizagem e praticar Matemática, já foram os que manifestaram maior alteração com a frequência em várias formações. Também se evidencia o TPK2, relacionado com a ideia que os docentes possuem como capazes de integrar o uso do QI na aprendizagem dos alunos, como o item com maior média e menor DP no caso dos docentes terem frequentado formação.

Graficamente as estatísticas descritivas do Quadro 4 podem ser visualizadas num diagrama de extremos e quartis (Gráfico), denotando uma diminuição da dispersão dos resultados e um aumento das médias de cada item do conhecimento 


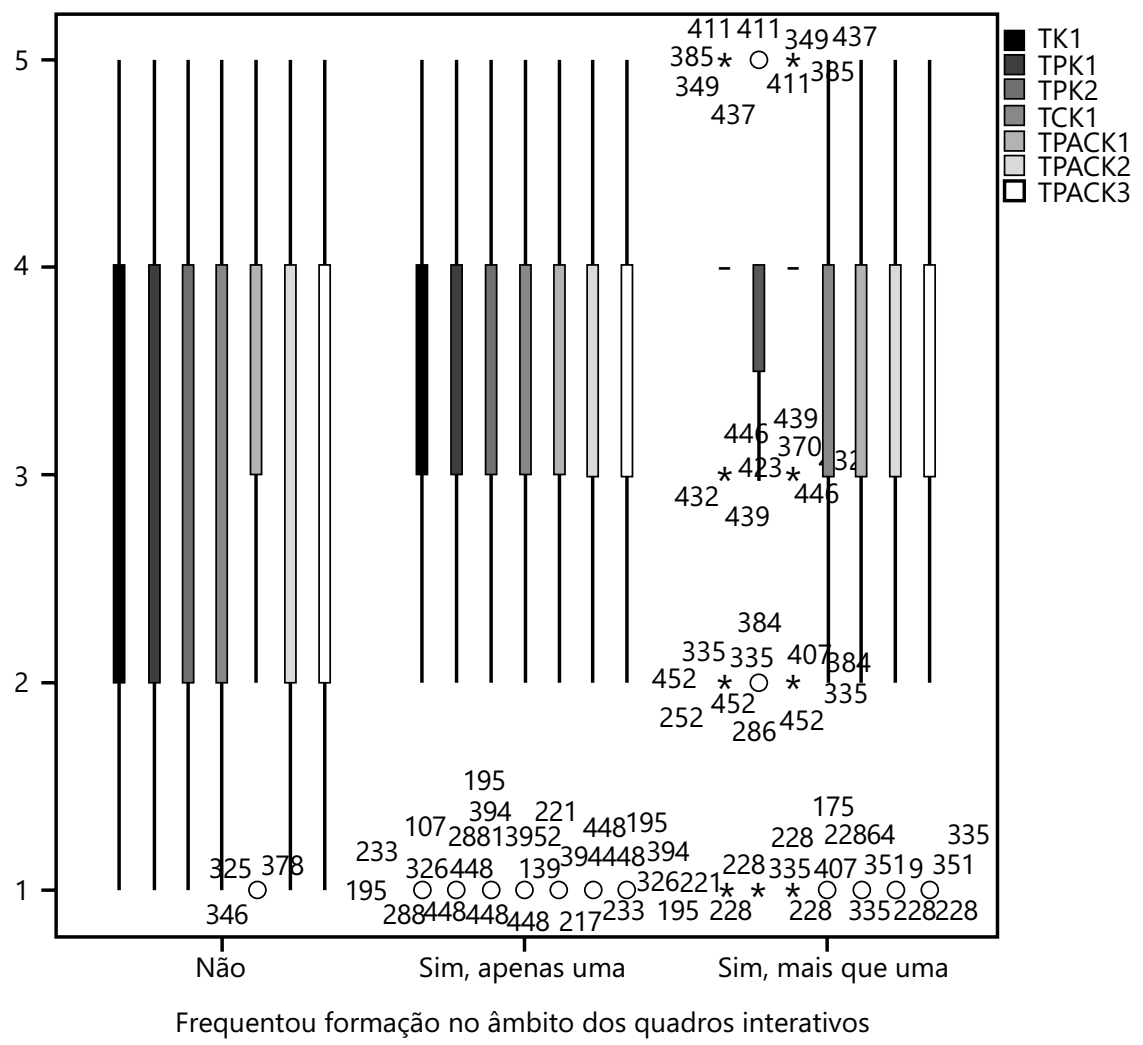

Fonte: Dados da pesquisa (2015).

Gráfico. Diagrama de extremos e quartis das respostas obtidas a cada item do conhecimento tecnológico dos professores em QI.

tecnológico dos docentes, à exceção do TPACK1, relativamente à frequência ou não em formação em QI no âmbito do PTE. No caso de os docentes terem frequentado mais que uma formação, os itens com menor dispersão e maior média são o TK1 e o TPK2.

Depois de considerarmos os efeitos da frequência de formação sobre QI no conhecimento tecnológico dos docentes de Matemática é possível afirmar que a formação teve um efeito estatisticamente significativo $(p<0,001)$ sobre TK1, TPK1, TPK2, TCK1, TPACK2 e TPACK3 (Quadro 5). Verificou-se, através do teste de Levene, que não havia homogeneidade de variância, condição necessária para a aplicação da ANOVA, o que resultou na utilização do teste não paramétrico, o teste de Kruskal-Wallis. 
Quadro 5. Teste Kruskal-Wallis em que a variável de agrupamento foi a frequência em formação no âmbito de QI.

\begin{tabular}{|l|c|c|c|c|c|c|c|}
\hline & TK1 & TPK1 & TPK2 & TCK1 & TPACK1 & TPACK2 & TPACK3 \\
\hline Qui-quadrado & 37,817 & 45,169 & 27,640 & 37,748 & 2,854 & 38,951 & 25,959 \\
\hline df & 2 & 2 & 2 & 2 & 2 & 2 & 2 \\
\hline Sig. & 0,000 & 0,000 & 0,000 & 0,000 & 0,240 & 0,000 & 0,000 \\
\hline
\end{tabular}

QI: quadro interativo; TK: Conhecimento Tecnológico; TPK: Conhecimento Tecnológico Pedagógico; TCK: Conhecimento Tecnológico de Conteúdo; TPACK: Conhecimento Tecnológico Pedagógico de Conteúdo.

Fonte: Dados da pesquisa (2015).

Quanto à questão aberta do questionário sobre a capacidade de adaptação que os docentes de Matemática possuem relativamente ao uso das tecnologias que aprenderam na formação realizada no âmbito do PTE a diferentes atividades de ensino de Matemática, obteve-se 241 respostas, das quais $45,2 \%$ foram positivas.

Dos 109 professores que concordaram com a afirmação, 37 não apresentaram qualquer justificação, 29 referiram que esta capacidade de adaptação da formação contínua está diretamente relacionada com a vontade dos docentes quererem integrar as TIC nas suas atividades, 15 salientaram a importância do conhecimento tecnológico adquirido na formação e a possível adaptação pedagógica, 10 descreveram a sua resposta no âmbito do desenvolvimento do TPACK Matemático, 10 referiram a motivação dos alunos e a possível adaptação aos conteúdos matemáticos e 8 deram mesmo exemplos de programas computacionais que utilizam nas aulas.

Os restantes docentes (132) discordaram da afirmação, tendo 32 referido como motivo pela não adaptação do uso das tecnologias que aprenderam na formação a atividades de ensino de Matemática a falta de recursos das escolas, 27 salientaram que a formação foi insuficiente, necessitando de mais horas, 17 referiram que esta adaptação dos conteúdos da formação às atividades em sala de aula é um processo difícil, 16 salientaram que a preparação de materiais toma demasiado tempo, nove consideraram que o uso das TIC poderá ser prejudicial à aprendizagem de Matemática por parte dos alunos, oito referiram a falta de tempo para preparar materiais, sete mencionaram a dificuldade em gerir a sala de aula quando recorrem às tecnologias, cinco acharam mesmo que o PTE em geral não tem qualquer utilidade para as aulas de Matemática, cinco mencionaram que o programa de Matemática é demasiado extenso, quatro reforçaram a falta de prática com o uso das TIC, sentindo-se desconfortáveis e dois não se sentem motivados para tal. 


\section{Discussão}

Relativamente à primeira questão de investigação, a opinião mais frequente dos docentes de Matemática relativa à perceção de autoeficácia do grau de competência na utilização das TIC na prática docente foi que possuem um nível bom, e mais de três quartos dos docentes utilizam as TIC nas suas aulas pelo menos com alguma frequência. Num estudo de caso com professores de Matemática realizado por Sampaio e Coutinho (2013c) chegou-se a conclusões semelhantes, isto é, que $75,0 \%$ dos docentes de Matemática usavam as TIC com frequência e que 85,0\% consideravam que possuíam um nível de competência em usar as TIC bom ou muito bom. Noutro estudo de caso com docentes desta área curricular, Vaz (2012) também concluiu que a maioria dos inquiridos se sentia capaz e utilizava o computador para elaborar testes, exames e fichas de avaliação, para registar e efetuar cálculos sobre a avaliação, enviar e-mails... Similarmente, Vicente e Melão (2009) concluíram que 82,6\% dos professores de Matemática inquiridos utilizava as TIC na sala de aula e $26,0 \%$ fazia uso das tecnologias com os seus alunos fora da sala de aula.

Quanto à utilização de QI na sala de aula há uma grande dispersão dos resultados e quase metade dos docentes $(47,7 \%)$ praticamente não os utiliza. Num estudo realizado por Sampaio e Coutinho (2013c) chegou-se a conclusões semelhantes, tendo-se concluído, ainda, que apenas $40,0 \%$ dos professores de Matemática usavam esta ferramenta educativa com frequência para promover a aprendizagem dos alunos. Ferreira e Meirinhos (2011), num outro estudo que realizaram, verificaram que apenas cerca de um terço dos docentes inquiridos utilizava o QI com frequência e que poucos professores produziam recursos próprios para serem usados com o QI. Vicente e Melão (2009), também num estudo com docentes de Matemática, averiguaram que a utilização do QI nas aulas não era muito elevada, já que $44,4 \%$ dos professores de Matemática, que já tinham tido um primeiro contato com o QI, nunca os utilizavam e a frequência de utilização foi considerada mesmo pouco regular.

Relativamente à terceira questão de investigação, verificaram-se grandes semelhanças entre os diferentes tipos de conhecimento tecnológico dos professores de Matemática inquiridos, sobre a utilização de QI, destacando-se apenas uma autoperceção mais elevada na capacidade de os integrar na aprendizagem dos alunos, num sentido de integração da tecnologia com a pedagogia em sala de aula. Os docentes de Matemática consideram, em geral, que possuem conhecimentos tecnológicos para usarem os QI nas aulas, salientando-se a compreensão de que os QI apresentam quer potencialidades quer limitações, podendo influenciar a aprendizagem dos alunos, a aquisição de habilidades técnicas para usar ferramentas 
dos QI e a capacidade de ensinar os alunos a usarem essas ferramentas. Não obstante, o item que apresentou menor média relaciona-se com a capacidade dos professores usarem as ferramentas de QI para proporcionarem aos alunos diferentes oportunidades de aprendizagem e praticar Matemática. Estando estes conhecimentos diretamente relacionados com o conteúdo matemático e não apenas com a tecnologia em si ou com questões pedagógicas sem interligação com a Matemática.

A literatura afirma que o uso das TIC nas aulas de Matemática não apresenta só aspetos positivos, as tecnologias também apresentam limitações (SAMPAIO; COUTINHO, 2013b), que quando não são consideradas até podem resultar em aprendizagens menos corretas (FERNANDES; VAZ, 1998) e se a planificação da aula não estiver bem estruturada, de modo a se tirar o máximo partido das ferramentas educativas, corre-se o risco de os resultados ficarem bastante aquém do esperado (SILVA, 2005). A utilização das TIC pode criar, "um ambiente de aula com mais movimento, mais ruído, mais sobressaltos e receios para o professor" (AMADO; CARREIRA, 2008, p. 287), o que implica um acréscimo das decisões que o professor deverá tomar quer na planificação das tarefas quer na sua implementação (SANTOS, 2000).

A utilização do QI no processo de ensino/aprendizagem não é suficiente por si para promover ambientes de aprendizagem interativos (SAMPAIO; COUTINHO, 2013a; WARWICK; HENNESSY; MERCER, 2011). Esta interatividade está sujeita à natureza das interações e do diálogo estabelecido na sala de aula entre professor/aluno e aluno/aluno (BEAUCHAMP; KENNEWELL, 2010).

Curiosamente, as aulas em que se evidenciaram mais contributos da utilização do quadro interativo não foram aquelas em que se alcançou um nível de interatividade mais elevado e em que foram utilizadas mais funcionalidades. Este resultado comprova que existem outros fatores, para além do recurso à tecnologia, que determinam o grau de interatividade que se estabelece na sala de aula, como é o caso do papel desempenhado pela professora ou o tipo de tarefa. (REIS, 2014, p. 155-156)

Quanto à relevância da formação no conhecimento tecnológico dos professores de Matemática em QI, proporcionada pelo PTE, constataram-se diferenças significativas em todos os itens à exceção da autoperceção dos professores sobre as potencialidades e as limitações dos QI no sentido de poderem influenciar a aprendizagem dos alunos. Por outro lado, os conhecimentos no âmbito tecnológico 
ou pedagógico e tecnológico foram os que manifestaram maior alteração com a frequência em mais que uma formação sobre QI.

Apesar dos estudos aqui apresentados terem salientado, em grande parte, as vantagens do uso do QI em contexto de sala de aula, é necessário realçar que há uma variação considerável quanto à utilização da ferramenta dentro dos departamentos das escolas. A introdução dessa tecnologia não permite, em si, transformar pedagogias existentes, sendo necessário, para tanto, haver um enfoque nas discussões entre os colegas sobre como o QI pode ser usado para apoiar, ampliar e transformar a prática docente. (SAMPAIO; COUTINHO, 2013a, p. 750)

Para se obter um nível relevante de integração das TIC na sala de aula há que ter em conta o papel do professor (GOODISON, 2002; SAMPAIO; COUTINHO, 2012). Neste estudo, verificou-se que a formação em QI foi relevante para melhorar os vários tipos de conhecimento tecnológico dos professores, mas como os docentes afirmaram, na questão aberta, a capacidade de adaptação da formação contínua às atividades de sala de aula está diretamente relacionada com a vontade dos docentes quererem integrar as TIC nas suas atividades. "Não podemos mais aceitar a premissa de que simplesmente por se colocar um QI numa aula de Matemática do ensino secundário se irá produzir melhores resultados em Matemática" (MILLER; GLOVER; AVERIS, 2008, p. 4).

A partir desta pesquisa, também se constatou que a frequência em mais que uma formação dentro do mesmo âmbito não diminuiu a dispersão dos resultados relativamente ao desenvolvimento do TCK ou do TPACK, aproximando apenas o conhecimento dos docentes relativamente ao TK e ao TPK. Ou seja, com a frequência em mais que uma formação os docentes desenvolveram as habilidades técnicas para usarem ferramentas de QI, sentem-se mais confiantes para ensinar os seus alunos a usarem essas ferramentas e sentem-se capazes de integrar o uso de QI na aprendizagem dos alunos, de um modo geral. No entanto, a integração da tecnologia com os conteúdos matemáticos continua a variar bastante de docente para docente. Os professores ainda referiram que a adaptação dos conteúdos da formação às atividades das aulas é um processo difícil, a preparação de materiais toma demasiado tempo e não há tempo para os preparar. Um número considerável de docentes ainda referiu a falta de recursos das escolas apesar dos esforços governamentais com o PTE. 
Um estudo realizado no âmbito do Observatório do PTE (LOPES, 2010) com o intuito de inventariar comportamentos digitais e recolher elementos significativos para a gestão desta medida do governo, que envolveu diretores, professores, encarregados de educação e alunos de algumas escolas de Portugal, evidenciou que os docentes utilizam frequentemente as TIC em ambiente educativo como a Internet, os computadores e os projetores multimédia, mas ainda se está longe de rentabilizar ao máximo todo equipamento tecnológico fornecido pelo PTE. Os docentes consideraram que ainda precisavam de reforçar os seus conhecimentos técnicos e a autoconfiança na utilização das TIC através da formação. A falta de atualização tecnológica dos docentes pode originar constrangimentos com os seus alunos, mais hábeis com variados dispositivos informáticos (AREA, 2010).

Entretanto como já passaram alguns anos desde a investigação de Lopes (2010), neste estudo os docentes de Matemática já manifestaram ter frequentado formação no âmbito do PTE e terem adquirido mais conhecimentos técnicos sobre como usar QI. "Os professores necessitam de tempo para desenvolver a sua fluência tecnológica, aplicar princípios pedagógicos aos materiais disponíveis ou para o desenvolvimento de materiais, e em seguida para incorporar o QI perfeitamente no seu ensino" (MILLER; GLOVER; AVERIS, 2005, p. 16).

No estudo realizado por Lopes (2010) também se tinha concluído que a utilização das TIC nas aulas implica um suplemento de trabalho para o docente e de tempo para conseguir preparar recursos digitais. Referindo ainda que há uma dificuldade acrescida na gestão da sala de aula e que uma quantidade considerável de salas das escolas portuguesas ainda não estavam suficientemente preparadas para o trabalho com recurso às TIC.

Como verificamos, "desde o reconhecimento de que a tecnologia poderá ser ou não útil no processo de ensino/aprendizagem da Matemática até à efetiva integração da mesma na sala de aula e sua avaliação, há um longo caminho a percorrer" (SAMPAIO; COUTINHO, 2012, p. 105). Através da formação contínua os docentes realmente melhoraram a sua autoperceção de utilização das TIC, em particular do QI, no sentido de desenvolverem o conhecimento tecnológico de todos os tipos, mas os docentes necessitam de tempo para mudarem as suas práticas em contexto de sala de aula (GUSKEY, 2002; SAMPAIO; COUTINHO, 2013c).

\section{Conclusão}

Pela implementação das políticas públicas do PTE em Portugal, como grande projeto do governo, que envolveu quase todas as escolas públicas do país e um esforço económico nunca visto em Portugal, torna-se importante refletir sobre o 
seu impacto na prática letiva dos docentes. Neste estudo pretendeu-se conhecer a autoperceção dos docentes de Matemática sobre o conhecimento tecnológico que possuem na utilização de QI e qual a frequência de utilização dos mesmos nas suas aulas, para além de tentar perceber se a frequência de formação, no âmbito do PTE, provocou diferenças nesse conhecimento tecnológico em QI.

As conclusões do estudo são que os professores de Matemática consideram que possuem um nível bom em TIC e usam ferramentas tecnológicas com frequência nas suas aulas. No entanto, no caso particular dos QI há uma grande dispersão dos resultados e poucos usam esta ferramenta educativa com frequência com os seus alunos. Os docentes de Matemática consideram ser detentores de conhecimento tecnológico sobre QI, mas ainda é visível uma grande dispersão dos resultados e $\mathrm{o}$ item que os docentes sentem maior necessidade de melhoria relaciona-se com a capacidade de usar as ferramentas de QI para proporcionar aos alunos diferentes oportunidades de aprendizagem e praticar Matemática. Finalmente, a frequência em formação contínua trouxe melhorias significativas no conhecimento tecnológico dos docentes de Matemática em QI, mas ainda há um longo caminho a percorrer para o desenvolvimento do TPACK Matemático.

De futuro seria pertinente que se desenvolvessem outras investigações sobre o impacto do PTE nas escolas portuguesas. Como estão a ser usadas as TIC em contexto de sala de aula? Há diferenças entre disciplinas? Quais as alterações metodológicas dos docentes? Quais as repercussões quanto à aprendizagem dos alunos? 


\title{
Mathematics teachers' technological knowledge about interactive whiteboards according to public policies of lifelong training
}

\begin{abstract}
The Education Technological Plan was a major program of technological improvement in Portuguese schools. It was composed by three axes: Technology, Content and Training. In this framework, a research was made on the effect of these public policies on teachers' daily practices using interactive whiteboards (IW). A questionnaire was applied to 453 math teachers in Portugal. It was concluded that teachers consider they have a good level of ICT knowledge and they frequently use technological tools in classes. They also consider they have technological knowledge on IW, but a widespread of its use can still be seen. Teachers feel a great need to improve their ability to use the IW tools in order to give students different opportunities when learning and practicing mathematics. Teachers upgraded their technological knowledge on IW tools by participating in lifelong training under these policies. Nevertheless, there is still a long way to go to improve Mathematics TPACK.
\end{abstract}

Keywords: Interactive whiteboard. Mathematics TPACK. Lifelong training. Public policies.

\section{El conocimiento tecnológico de los profesores de Matemáticas sobre la pizarra interactiva según las políticas públicas de formación continua}

\section{Resumen}

El Plan Tecnológico de Educación fue el mayor programa de modernización tecnológica de las escuelas portuguesas, siendo compuesto por tres ejes: tecnología, contenidos y formación. Presentamos un estudio sobre el impacto de estas políticas públicas en las actividades de los profesores sobre el uso de pizarras interactivas (PI). Se aplicó un cuestionario a 453 docentes de matemáticas de Portugal. Se concluyó que todos los profesores consideran que poseen un buen nivel en TIC y usan, frecuentemente, en sus clases, herramientas tecnológicas. Consideran tener conocimiento tecnológico sobre PI, pero aún es visible una gran dispersión de uso. Los docentes sienten mayor necesidad de perfeccionamiento de la capacidad de usar las herramientas de las PI a fin de proporcionar a los alumnos diferentes oportunidades de aprendizaje y practicar matemáticas. La frecuencia en formación continua en el ámbito de estas políticas ha traído mejoras significativas en el conocimiento tecnológico de los profesores de matemáticas sobre esta herramienta, pero aún queda mucho camino por recorrer para el desarrollo del TPACK Matemático.

Palabras clave: Pizarra interactiva. TPACK Matemático. La formación continua. Políticas públicas. 


\section{Referências}

AMADO, N.; CARREIRA, S. Utilização pedagógica do computador por professores estagiários de Matemática: diferenças na prática da sala de aula. In: CANAVARRO, A., MOREIRA, D.; ROCHA, M. (Org.) Tecnologias e educação matemática. Vieira de Leiria: SEM/SPCE, 2008. p. 286-99.

AREA, M. El proceso de integración y uso pedagógico de las TIC en los centros educativos: un estudio de caso. Revista de Educación, v. 352, p. 77-97, 2010.

BEAUCHAMP, G.; KENNEWELL, S. Interactivity in the classroom and its impact on learning. Computers \& Education, v. 54, p. 759-66, Apr. 2010. doi:10.1016/j.compedu.2009.09.033

DGEEC - DIREÇÃO-GERAL DE ESTATÍSTICAS DA EDUCAÇÃO E CIÊNCIA. Estatísticas da educação 2012/2013. Lisboa: DGEEC, 2014.

FERNANDES, J.; VAZ, O. Porquê usar tecnologia nas aulas de Matemática? Boletim da SPM, n. 39, p. 43-55, out. 1998.

FERREIRA, A.; MEIRINHOS, M. Utilização de quadros interactivos multimédia numa escola do ensino básico. In: CONFERÊNCIA IBÉRICA: INOVAÇÃO NA EDUCAÇÃO COM TIC, 2011, Bragança. Anais... Bragança: Instituto Politécnico de Bragança, 2011. p. 337-51.

GOODISON, T. ICT and attainment at primary level. British Journal of Educational Technology, v. 33, n. 2, p. 201-11, Mar. 2002. doi:10.1111/1467-8535.00253

GUSKEY, T. Professional development and teacher change. Teachers and Teaching: Theory and Practice, v. 8, n. 3/4, p. 381-91, 2002. doi:10.1080/135406002100000512

KOEHLER, M.; MISHRA, P. What happens when teachers design educational technology? The development of technological pedagogical content knowledge. Journal Educational Computing Research, v. 32, n. 2, p. 131-52, Mar. 2005. doi:10.2190/0EW7-01WB-BKHL-QDYV

LOPES, H. Relatório de resultados do inquérito aos adultos sobre o Plano Tecnológico da Educação. Lisboa: Centro de Estudos dos Povos e Culturas de Expressão Portuguesa - Universidade Católica Portuguesa, 2010. 
MILLER, D.; GLOVER, D.; AVERIS, D. Presentation and pedagogy: the effective use of interactive whiteboards in mathematics lessons. In: BRITISH CONGRESS OF MATHEMATICS EDUCATION, 6. 2005, London. Proceedings... London: British Society for Research into Learning Mathematics, 2005. p. 105-12. Disponível em $<$ http://bit.ly/ bsrlm25iwbmiller>. Acesso em: 9 ago. 2014.

. Enabling enhanced mathematics teaching with interactive whiteboards: final report for the National Centre for Excellence in the Teaching of Mathematics. Staffordshire: Keele University, 2008. Disponível em: <http://bit.ly/iwbreport>. Acesso em: 9 ago. 2014.

MISHRA, P.; KOEHLER, M. Technological pedagogical content knowledge: a framework for teacher knowledge. Teachers College Record, v. 108, n. 6, p. 1017-54, 2006.

NIESS, M. Guest editorial: preparing teachers to teach mathematics with technology. Contemporary Issues in Technology and Teacher Education, v. 6, n. 2, p. 195-203, 2006.

PALIS, G. R. O conhecimento tecnológico, pedagógico e do conteúdo do professor de Matemática. Educação Matemática Pesquisa, v. 12, n. 3 , p. 432-51, 2010.

REIS, C. O quadro interativo e o desenvolvimento da interação dialógica na aula de Matemática. 2014. 185 f. Tese (Mestrado em Ciências da Educação) - Universidade de Évora, Évora, 2014.

SAMPAIO, P. A. S. R.; COUTINHO, C. P. Ensinar matemática com TIC: em busca de um referencial teórico. Revista Portuguesa de Pedagogia, v. 46, n. 2, p. 91-109, 2012.

. Quadros interativos na educação: uma avaliação a partir das pesquisas da área. Educação e Pesquisa, v. 39, n. 3, p. 741-56, 2013a. doi:10.1590/S1517-97022013000300012

Ensinar com tecnologia, pedagogia e conteúdo.

Revista Paidéi@, v. 5, n. 8, jul.2013b. Disponível em:

$<\mathrm{http}$ ://revistapaideia.unimesvirtual.com.br/index.php?journal= paideia\&page $=$ article \&op=view\&path[] $=304 \&$ path[] $=314>$.

Acesso em: 9 ago. 2014 
SAMPAIO, P. A. S. R.; COUTINHO, C. P. Teach mathematics with technology: put into practice a theoretical framework. In SOCIETY FOR INFORMATION TECHNOLOGY \& TEACHER EDUCATION INTERNATIONAL CONFERENCE, 2013, Chesapeake, Proceedings... Chesapeake, VA: AACE, 2013c. p. 4852-7. Disponível em <http://www. editlib.org/p/48897.0>. Acesso em: 9 ago. 2014.

SANTOS, E. O computador e o professor: um contributo para o conhecimento das culturas profissionais dos professores. Quadrante, v. 9, n. 2, p. 55-81, 2000.

SILVA, R. Gestão da aprendizagem e do conhecimento. In: SILVA, R.; SILVA, A. (Org.). Educação, aprendizagem e tecnologia: um paradigma para professores do Século XXI. Lisboa: Edições Sílabo, 2005. p. 41-65.

VAZ, J. Formação contínua de professores em tic: auto-eficácia e utilização. 2012. 53 f. Tese (Mestrado em Gestão de Sistemas de E-Learning) - Universidade Nova de Lisboa, Lisboa, 2012.

VICENTE, C.; MELÃO, N. A adopção do Quadro Interactivo pelos professores de matemática do $3^{\circ} \mathrm{CEB}$ : um estudo empírico nas escolas da Guarda. Educação, Formação \& Tecnologias, v. 2, n. 2, p. 41-57, nov. 2009.

WARWICK, P.; HENNESSY, S.; MERCER, N. Promoting teacher and school development through co-enquiry: developing interactive whiteboard use in a 'dialogic classroom'. Teachers and Teaching, v. 17, n. 3, p. 303-24, 2011. doi:10.1080/13540602.2011.554704

\section{Informações da autora}

Patricia Alexandra da Silva Ribeiro Sampaio: Doutoranda na Universidade do Minho. Professora de Matemática do ensino secundário, licenciada em Matemática pela Universidade do Minho (Portugal) e mestre em Tecnologia Educativa pela mesma universidade. Formadora reconhecida pelo Conselho Científico-Pedagógico da Formação Contínua e pelo Instituto do Emprego e Formação Profissional. Participou na equipa de investigação CIEd como colaboradora nos projetos de investigação e aprendizagem, formação e investigação na web. Atualmente é bolsista da Fundação para a Ciência e Tecnologia com um projeto sobre a integração da tecnologia no ensino da Matemática, na área das Ciências da Educação. Contato: patisampaio@gmail.com 